Brit. J. Ophthal. (1958) 42, 54.

\title{
BURIED ORBITAL IMPLANT WITH EXTRACONJUNCTIVAL EXTENSION*
}

BY

\author{
STANISLAW GEBERTT \\ Aberdeen
}

THE diversity of types of orbital implants suggests that the ideal one is still to be found. In the present stage of our knowledge it appears that the best retained are those which are completely buried. On the other hand the movements of the artificial eye are more natural if there is direct contact between implant and prosthesis.

Work on the implant described below was begun in 1953 at the Coventry and Warwickshire Hospital and continued at the Aberdeen Royal Infirmary.

To the two essential qualities of an orbital implant, good retention and satisfactory cosmetic result, are added comparative simplicity in use and the possibility of using the model in every case of enucleation, including those of suspected intra-ocular neoplasm.

\section{Construction}

The implant consists of a main body made of transparent acrylic material, perforated cross-wise horizontally, and having four gold wire prongs projecting forward to form a rectangle. The prongs serve as a support and spring lock for a round acrylic platform, containing in its front surface a rectangular hole into which fits the peg of the prosthesis. One side of the body and the corresponding side of the platform are marked with pink acrylic to ensure the proper fitting of the spring lock (Figs 1, 2, and 3).
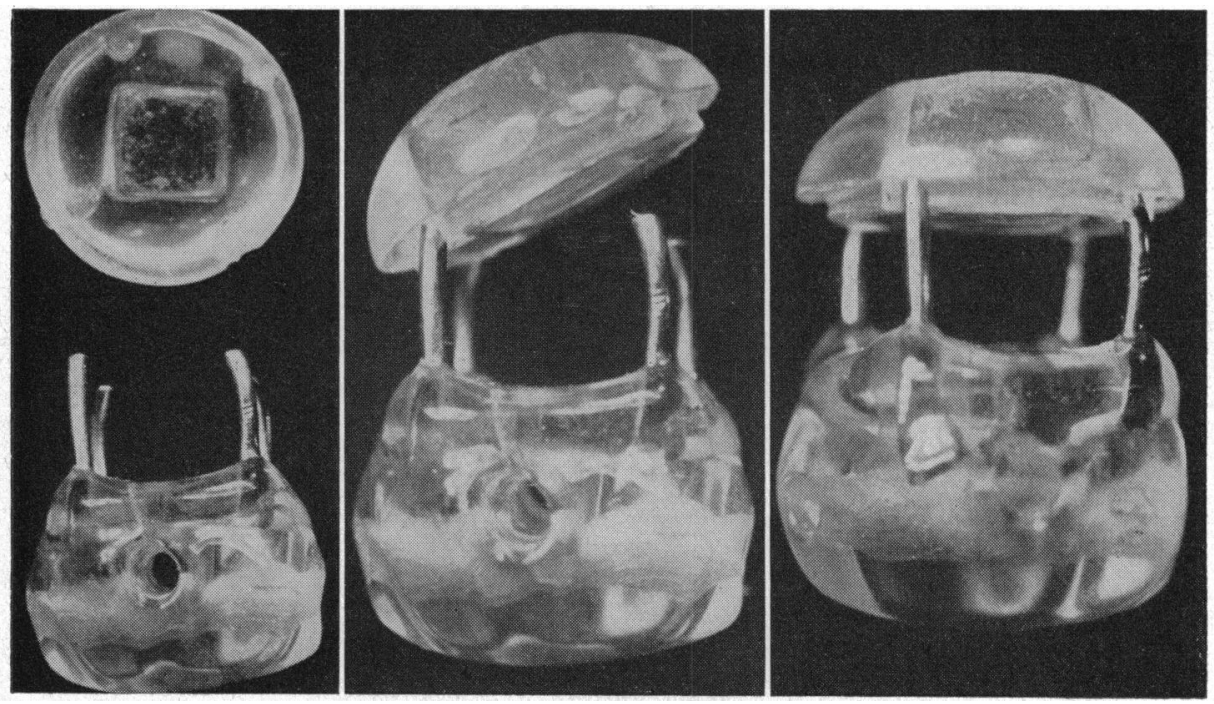

Figs. 1-3.-Fitting the extra-conjunctival section to the orbital implant.

* Received for publication March 9, 1957. 
The main body is completely buried, the gold wires project forward through the slits in Tenon's capsule and conjunctiva, and the top platform is thus extraconjunctival.

\section{Technique}

The "Stellon" technique is used throughout in preparing the acrylic, and the implant is sterilized by immersion in solution cetrimide B.P. for 12 hours.

Instruments.-Speculum. No. 15 Bard-Parker knife. Squint scissors. Excision scissors. Conjunctival forceps, one into two and two into three teeth. Untoothed forceps. Two strabismus hooks. Needle holder. Twelve mosquito forceps. Tool for "pressing in" acrylic platform. Ten black silk sutures Size 1: eight single-ended, and two double-ended with curved needles No. 4. Six black braided silk sutures No. 00 with eyeless corneo-scleral needle. One catgut suture No. $3 / 0$ with double eyeless curved needle No. 7 .

Operation:-When general anaesthesia has been effected, a speculum is inserted. The conjunctiva is cut close to the limbus around its perimeter and well undermined. Four retention sutures Size 1 are passed diagonally and clamped with mosquito forceps to the towels. Tenon's capsule is opened between the rectus muscles. The internal rectus is lifted on the strabismus hook, the edges and surface are cleaned with a swab, and if necessary it is dissected for $8 \mathrm{~mm}$.

A double-ended braided silk suture is passed through the muscle $2 \mathrm{~mm}$. behind its insertion and transversely to its long axis. The muscle is divided close to the sclera. The needles of the suture are passed medially through the towel and clamped with mosquito forceps. The same procedure is repeated on the remaining rectus muscles, leaving 1-mm. stump of external rectus (Fig. 4).

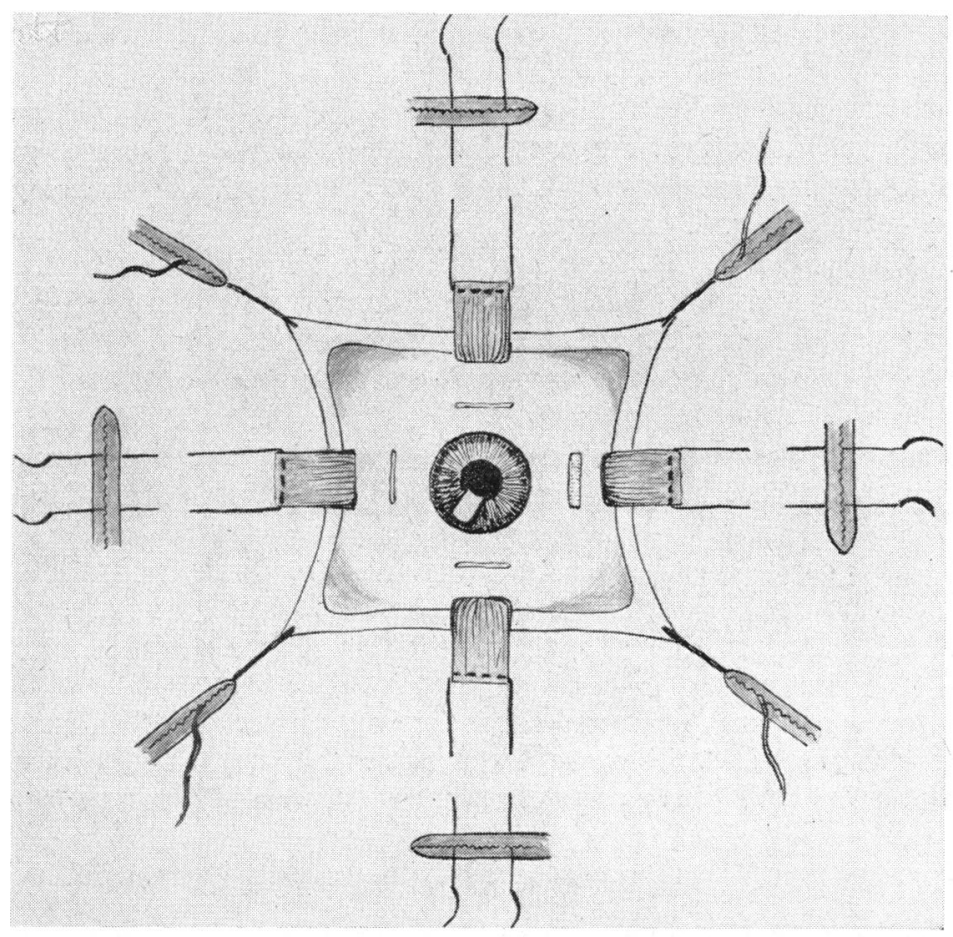

Fig. 4.-Conjunctiva retracted. Rectus muscles detached with their sutures sewn through the towels and clamped. 
The inferior and superior oblique muscles are divided, and the eye is proptosed and seized with fixation forceps by the external rectus stump. The excision scissors are introduced and the enucleation is completed in the usual way.

After haemostasis has been effected by a pressure pack, Tenon's capsule is secured with four retention sutures Size 1, one opposite each rectus muscle (Fig. 5). The sutures, weighted with mosquito forceps, are laid on the towels.

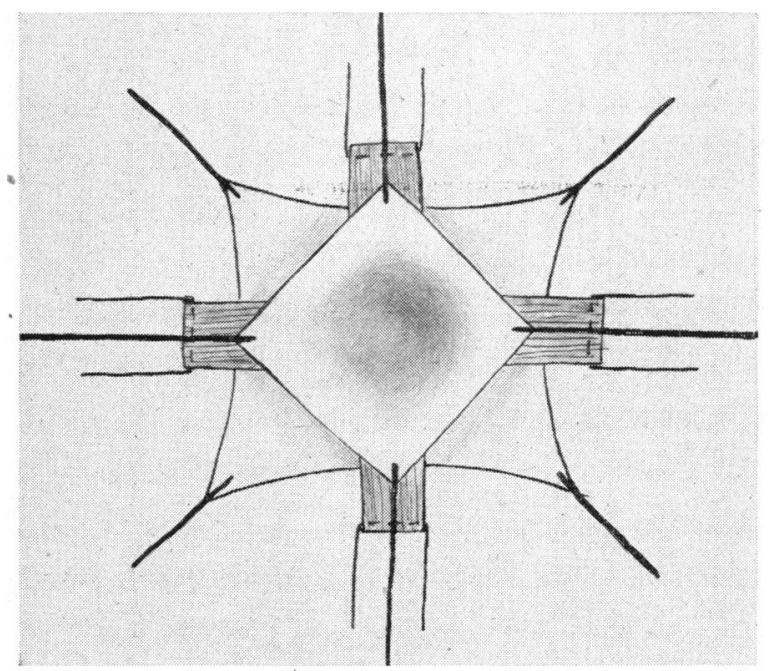

Fig. 5.-Tenon's capsule retracted.

The body of the implant, with the platform removed, is threaded with two double-ended Size 1 sutures (straight needle used and then replaced by a curved No. 4 needle).

The implant, with the side marked pink pointing nasally, is held above the socket by an assistant and its sutures are passed through Tenon's capsule from within outwards and forward, reaching about $1.5 \mathrm{~cm}$. deep, each needle being opposite each rectus muscle (Fig. 6, opposite).

The above process is facilitated by lifting each mosquito forceps retaining the capsule in turn while an assistant exercises a gentle pull on the muscle concerned. Care should be taken that all four needles pierce Tenon's capsule at the same level.

The implant is placed in proper position by a pull on its sutures which then lie over the rectus muscles.

A braided silk suture is passed purse-string-like through the edge of Tenon's capsule, the retaining sutures are removed, and the braided suture is drawn and tied, and the ends cut short.

To discern the gold prongs through Tenon's capsule, the implant is raised forward by a pull on its sutures. Four 1-mm. slits are cut to expose the wires, and two diagonally opposite wires are secured with just the tips of a pair of mosquito forceps.

The horizontal retaining suture of the implant is tied over its centre, then each needle is passed through Tenon's capsule above and below the knot, and the ends tied with the corresponding ends of the sagittal retaining suture and are cut short (Fig. $7 a, b$, opposite).

The double-ended catgut suture is then passed sagittally through $4 \mathrm{~mm}$. of tissue 

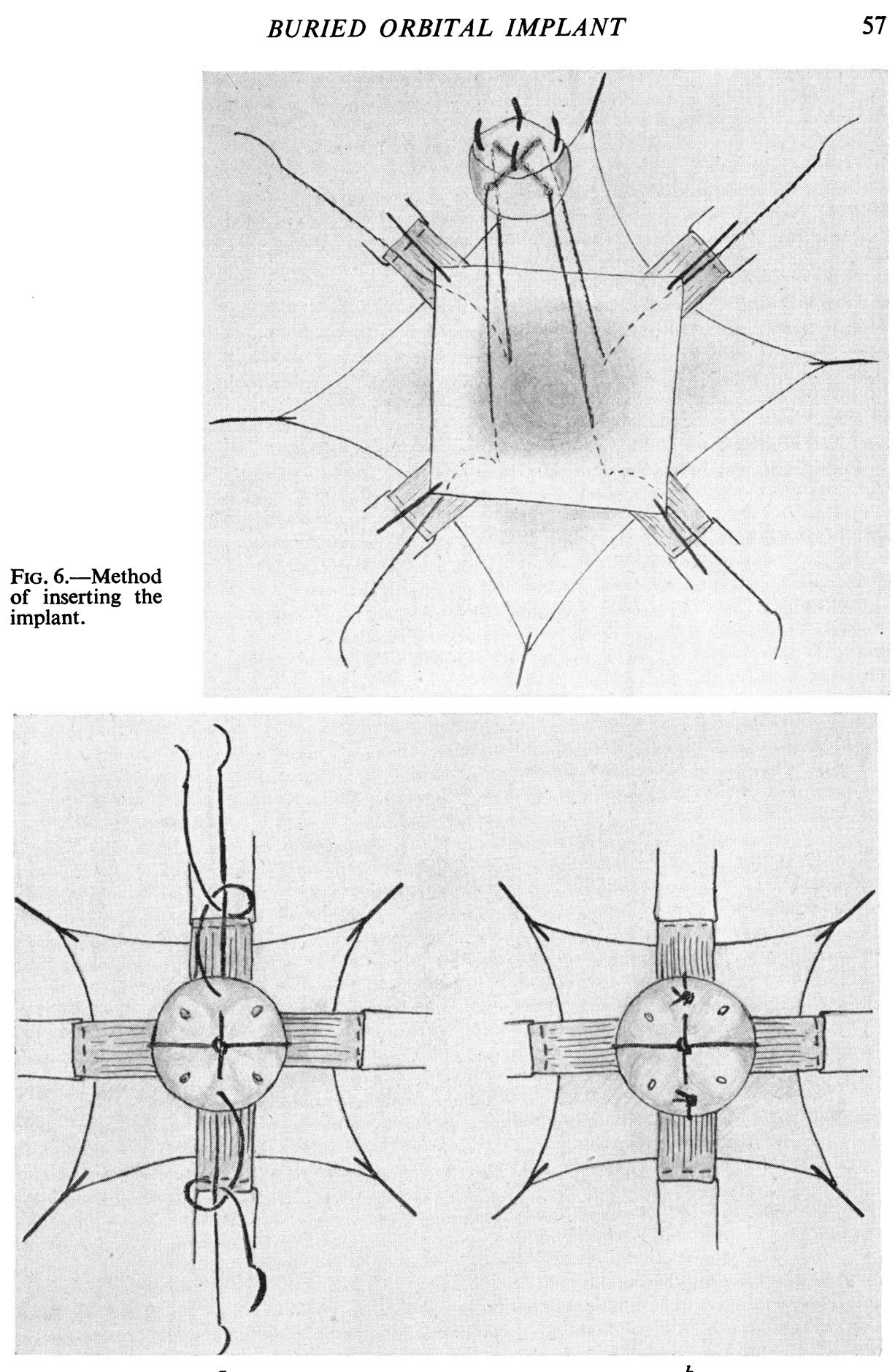

${ }^{a}$ FIG. $7(a, b)$.-Tying the sutures of the implant. 
in between the gold wires. By pulling on the muscle-retaining sutures, the ends of the internal and external rectus muscles are brought across over the implant, one above the other. The ends of the catgut suture are threaded through both muscles $2 \mathrm{~mm}$. behind the transverse silk sutures and tied on top. The ends of the silk sutures are cut short (Fig. 8).

The same process is repeated with the superior and inferior rectus muscles, and after being tied the cutgut suture is cut short.

A braided silk suture is passed purse-string-like through the edge of conjunctiva.

One at a time, the conjunctival retaining sutures are brought over the prongs, $1-\mathrm{mm}$. slits are cut through the conjunctiva, and each protruding wire is secured with mosquito forceps.

The conjunctival retaining sutures are then pulled out and the braided silk suture is tied in the centre.

The acrylic platform is fitted on the "pressing in" tool (Fig. 9) and secured in between the gold prongs, the side marked pink pointing nasally.

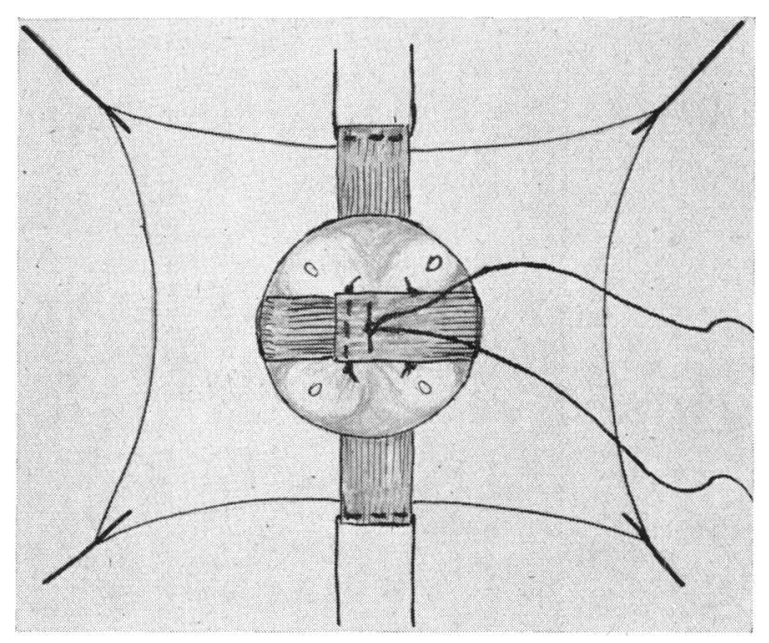

Fig. 8. - Internal and external rectus muscles tied on top of the implant.

Mosquito forceps attached to the wires help to apply counterpressure and to ease the spring lock. After the pressing tool has been removed the forceps are released (Fig. 10, opposite).

An antibiotic ointment is inserted into the conjunctival sac, and jelonet gauze dressing applied with only moderate pressure. The bandages are left undisturbed for $48 \mathrm{hrs}$.

Post-operative Course.-The post-operation reaction may occasionally be severe, with chemosis completely covering the implant. The socket is irrigated with normal saline two or three times daily, and by the end of first week the swelling should be negligible. A perforated shell is fitted between the tenth and fourteenth day and in 6 weeks the socket is ready for the proper prosthesis. 


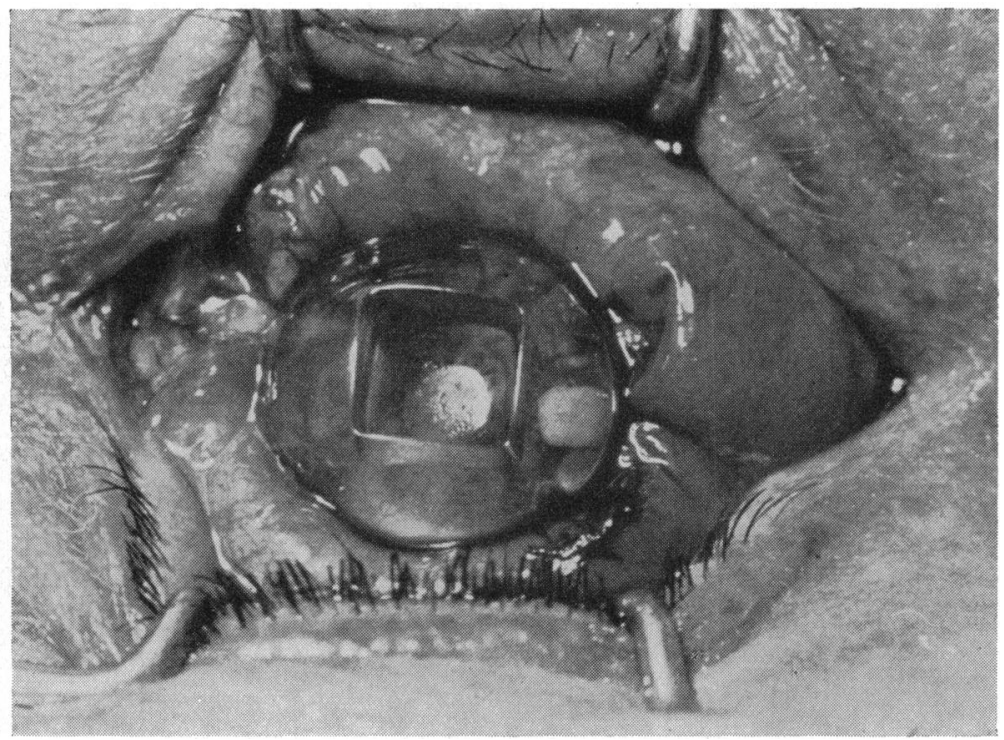

Fig. 10.-Operation completed.

Results

In all thirteen implants have been inserted. Two had to be removed within 3 weeks, one because of faulty operating technique, the other one because of a very severe reaction complicated by secondary infection.

Of the other eleven cases, four were completed in 1953, four in 1954, and three in 1955. All are still satisfactory.

\section{Future Developments}

Two possible improvements of the present model may be suggested:

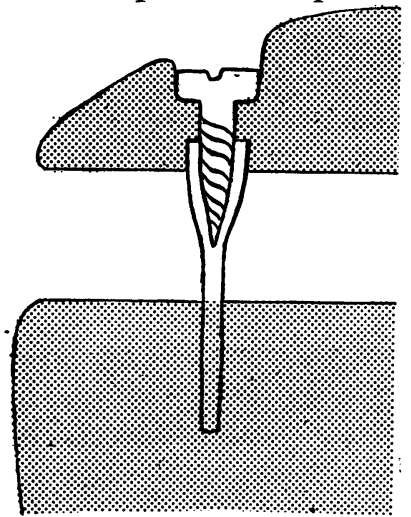

FIG. 11.-Alternative method of connecting the platform.

(1) The spring lock for securing the platform is by no means easy to operate, and an alternative method is shown in Fig. 11.

(2) The platform might with advantage incorporate a magnet.

With our limited engineering resources it has not yet been possible to produce an implant to these specifications.

I wish to express my thanks to Dr. Dorothy A. Campbell and Dr. Beatrix B. Law for their help and encouragement, to Mr. A. Gardiner for making the implants, to Mr. W. A. Rowley for fitting the prostheses, to Mr. R. Drummond for the photographs, and to my wife for the drawings.

\section{REFERENCES}

Choyce, D. P. (1952). Brit. J. Ophthal., 36, 123.

GeberTT, S. (1952). Trans. ophthal. Soc. U.K., 72, 585. 